\section{A percepção de pacientes e familiares sobre os resultados do tratamento em serviços de saúde mental}

\author{
Perceptions by patients and families towards \\ treatment outcomes in mental health services
}

\footnotetext{
1 Departamento de Psicologia, Universidade Federal de São João del-Rei, São João del-Rei, Brasil.

2 Departamento de Ciências Exatas, Universidade Federal de Lavras, Lavras, Brasil.

Correspondência

C. S. Costa

Laboratório de Pesquisa em Saúde Mental, Departamento de Psicologia, Universidade Federal de São João del-Rei. Praça Dom Helvécio 74, Campus Dom Bosco, São João del-Rei, $M G$

36300-000, Brasil.

cecilia.sico@gmail.com
}

\section{Abstract}

The need to evaluate treatment outcomes in mental health services from the user's perspective has been highlighted in the literature. The objective of this study was to compare the perceptions of patients and their families regarding treatment outcomes, in terms of perceived changes in the patient's life. The Perception of Change Scale was applied to 100 psychiatric patients that used public mental health services and 100 family members. The results showed a high percentage of agreement between patients' and families' perceptions of change on most items in the scale and on the open-ended questions. The results confirmed previous studies comparing patients' and families' satisfaction with other aspects of services. They also attest to the validity of patients' responses in the evaluation of treatment outcomes in mental health services.

Mental Health; Health Services; Treatment Outcome
Cecília Silva Costa 1

Marina Bandeira 1

Rita Laura Avelino Cavalcanti 1

João Domingos Scalon 2

\section{Introdução}

A avaliação sistemática das práticas e dos serviços de saúde mental é um procedimento recente, recomendado pela Organização Mundial da Saúde (OMS) 1. O processo de avaliação deve envolver os diferentes níveis dos serviços, ou seja, a sua estrutura, processo e resultados ${ }^{2}$. Além disso, a abordagem avaliativa recomendada inclui a participação do conjunto das pessoas envolvidas nestes serviços, a saber, pacientes, familiares e profissionais, para que a avaliação possa dar conta da complexidade do problema 1,3,4.

A literatura da área tem ressaltado cada vez mais a importância da participação dos usuários (pacientes e familiares), como parte integrante e necessária, no processo de avaliação da qualidade dos serviços de saúde mental 5,6, principalmente quando se trata de avaliar as suas percepções sobre os resultados do tratamento, através de escalas de medida validadas 5,7,8,9. Até o momento, o principal indicador de qualidade dos serviços comunitários de saúde mental, que envolve a percepção dos usuários, tem sido as medidas de satisfação ${ }^{5}$. No entanto, estas medidas são globais e abordam diferentes aspectos dos serviços, sem focalizar especificamente os resultados do tratamento, em termos das mudanças obtidas na vida dos pacientes 5,10.

Mercier et al. 5 destacaram a importância de se incluir a avaliação da percepção dos pacientes, especificamente sobre os efeitos do tratamento. 
Para isto, estes autores propuseram uma nova medida, que avalia a percepção de mudanças ocorridas na vida do paciente, de forma a identificar em quais aspectos de sua vida ocorreram melhora, piora ou não ocorreram mudanças, em função do tratamento recebido. Este novo indicador aponta, portanto, as dimensões da vida do paciente onde o tratamento atingiu seus objetivos e aquelas que necessitam de novas intervenções terapêuticas, para que ocorra uma melhora perceptível, fornecendo assim uma informação valiosa aos profissionais do serviço. Informações pontuais deste tipo contribuem para atingir melhores resultados do tratamento dos pacientes, promovendo a qualidade dos serviços.

Apesar das recomendações sobre a necessidade de avaliação dos serviços sob múltiplas perspectivas 1,11 , os estudos tendem a incluir apenas a percepção dos pacientes, quando o fazem. Há poucos estudos, na literatura da área, que incluem a perspectiva de ambos, pacientes e familiares. Fisher et al. 12 apontam que pacientes e familiares tendem a expressar opiniões diferentes com relação às suas prioridades sobre os resultados do tratamento, evidenciando a importância de se incorporar múltiplas perspectivas no processo de planejamento dos serviços. Segundo Holley et al. 13, os pontos em que houver acordo entre as opiniões podem constituir uma base sólida para o atendimento das necessidades dos pacientes, enquanto os pontos de desacordo podem fornecer informações para o planejamento estratégico, a definição de prioridades de serviço e a identificação das áreas que necessitam reformulações nas intervenções terapêuticas e medicamentosas, adaptando-as às necessidades dos pacientes e dos seus familiares, em função dos efeitos do tratamento.

Estudos comparativos das perspectivas de pacientes, familiares e profissionais dos serviços de saúde mental são raros. Em uma busca nas bases de dados do MEDLINE, LILACS e SciELO, foram encontrados nove estudos que compararam a percepção de, pelo menos, dois destes agentes a respeito de algum aspecto dos serviços. Apenas três destes estudos compararam a percepção dos pacientes e familiares quanto a: sua satisfação com os serviços 14,15 e seus conceitos de saúde, doença mental e assistência psiquiátrica 16. Um estudo avaliou a percepção dos pacientes, familiares e profissionais dos serviços sobre as prioridades dos resultados do tratamento e as prioridades dos serviços prestados 12 e outro estudo avaliou as opiniões dos três a respeito da mudança do tratamento psiquiátrico do hospital para a comunidade 13 .

No contexto brasileiro, estudos sobre a percepção de mudança pelos usuários em função do tratamento estão apenas começando, tendo sido realizados uma pesquisa de adaptação transcultural da escala proposta por Mercier et al. 5, em duas versões, uma para o paciente e outra para o familiar 17 e estudos para validação destes instrumentos de medida 18,19. Foram realizadas ainda duas pesquisas avaliando os fatores associados à percepção de mudanças em função do tratamento, a partir da percepção dos pacientes $20 \mathrm{e}$ da percepção dos familiares 21 . Não há, entretanto, nenhum estudo de comparação das percepções de mudanças dos pacientes e familiares. $\mathrm{O}$ presente estudo visa contribuir para suprir essa lacuna, investigando pontos de convergência e divergência entre as percepções de mudança dos pacientes e familiares, em função do tratamento recebido em serviços de saúde mental.

\section{Método}

\section{Participantes}

Participaram deste estudo, 100 pacientes psiquiátricos e os 100 familiares cuidadores destes pacientes. Os sujeitos eram atendidos nos Centros de Atenção Psicossocial (CAPS) de duas cidades de médio porte da região do campo das Vertentes, em Minas Gerais, Brasil, sendo que $50 \%$ da amostra de pacientes e familiares foram entrevistados em cada um deles. Apesar de serem pacientes atendidos em CAPS, eles estavam em regime ambulatorial nos serviços e, portanto, recebiam basicamente tratamento medicamentoso, com consultas médicas mensais.

A amostragem foi não probabilística e o tamanho da amostra foi limitado, devido à dificuldade em obter a participação de pares de pacientes e seus familiares e de preencher os critérios de inclusão e exclusão. Foram incluídos os pacientes com idade acima de 18 anos, de ambos os sexos, com diagnósticos de transtornos mentais severos e persistentes (Classificação Internacional de Doenças, 10a revisão-CID-10), que se tratavam na instituição há, pelo menos, um ano e que tinham um familiar cuidador que atendia aos critérios de inclusão e exclusão do estudo. Excluíram-se os pacientes que tinham alguma outra doença crônica grave, comorbidade neurológica ou dependência química, que estavam internados, em crise e que apresentavam dificuldade de compreender as questões dos instrumentos de avaliação. Para os familiares, os critérios de inclusão foram: ter idade acima de 18 anos, de ambos os sexos, serem indicados como principais cuidadores e ter frequência de contato com o paciente de, pelo menos, uma vez por semana. Foram eliminados aqueles familiares que 
não conseguiram responder aos instrumentos de avaliação ou que eram portadores de transtornos mentais severos e persistentes.

\section{Instrumentos de medida}

Utilizou-se a Escala de Mudança Percebida na versão dos pacientes (EMP-pacientes) e na versão dos familiares (EMP-familiares) e um questionário de avaliação das variáveis sócio-demográficas e clínicas. A escala EMP originou-se do Questionnaire of Perceived Changes, elaborado por Mercier et al. ${ }^{5}$. As duas versões brasileiras da escala foram obtidas através de estudos de adaptação transcultural 17 e estudos de suas propriedades psicométricas de validade e fidedignidade 18,19 . Ambas possuem os mesmos 19 itens que avaliam as mudanças percebidas na vida do paciente, do ponto de vista do paciente e do familiar. As alternativas de resposta, para cada item, se distribuem em uma escala tipo Likert de três pontos, sendo 1 - pior do que antes, 2 - sem mudança, 3 - melhor do que antes. As escalas possuem ainda uma questão aberta para avaliar se o tratamento recebido está ajudando o paciente a se sentir melhor, pedindo-se ao entrevistando que ele justifique a sua resposta. A escala original foi recentemente renomeada como Perceived Improvement Questionnaire (PIQ) por Perreault et al. 22 .

O questionário sociodemográfico e clínico foi utilizado para caracterização da amostra. Este questionário havia sido previamente testado em um estudo piloto, visando avaliar a adequação das questões para um bom entendimento pela população-alvo.

\section{Procedimento}

\section{- Coleta de dados}

As entrevistas foram realizadas por entrevistadores previamente treinados. A aplicação dos instrumentos foi realizada individualmente, em uma sala do serviço ou na casa dos respondentes, conforme a sua disponibilidade. A participação de todos foi voluntária.

\section{- Análise de dados}

Foram realizadas análises estatísticas com os dados de cada grupo separadamente (análise intragrupo) e análises comparativas entre os grupos de pacientes e familiares (análise intergrupos). As análises intragrupo incluíram a descrição das características da amostra, através de médias, desvios-padrão (DP) e porcentagens e a descrição dos escores de percepção de mudança, através da porcentagem de respostas de me- lhora, piora e ausência de mudança, aos itens da EMP. Uma análise da média de postos, pelo teste não paramétrico de Friedman, foi realizada para identificar, dentro de cada grupo, os itens que acarretaram uma maior percepção de mudança.

As análises intergrupos incluíram: (1) comparação das médias de postos de Friedman dos dois grupos, através do teste de correlação de Spearman, para verificar se havia correlações significativas entre estas duas distribuições de escores, e através do teste de Wilcoxon, para identificar quais itens apresentavam diferenças significativas entre as médias de postos, por pares de pacientes-familiares; (2) comparação das medianas dos escores dos dois grupos, usando o teste de análise das diferenças de Wilcoxon, para verificar se havia uma diferença estatisticamente significativa entre os escores de mudança percebida, dos pares de pacientes e familiares; (3) comparação dos escores brutos da percepção de mudança, realizada através de três análises: cálculo do coeficiente de correlação intraclasse (ICC) para cada par de escores brutos; análise das diferenças através da subtração dos escores dos pacientes dos escores dos familiares, para cada item da escala, obtendo-se a porcentagem de acordo e desacordo entre os pares de pacientefamiliar; análise estatística pelo coeficiente de contingência (CV), disponível na função Crosstabs do programa SPSS (SPSS Inc., Chicago, Estados Unidos), para investigar quais itens apresentavam uma distribuição de acordo e desacordo significativamente concordantes.

Para análise da primeira questão aberta da escala EMP dos pacientes e dos familiares, foi utilizada a técnica de análise de conteúdo, proposta por Bardin 23. Esta análise foi realizada a partir do método de dedução frequencial, em que cada aspecto abordado ao longo das entrevistas foi contabilizado, em termos da frequência que apareceu no total de entrevistas realizadas. Em seguida, foi realizada a análise categorial do tipo temática, em que os aspectos relatados foram classificados em categorias, de acordo com o seu conteúdo temático comum 23,24.

\section{- Considerações éticas}

O projeto desta pesquisa foi avaliado e aprovado pelas instituições psiquiátricas envolvidas e pelo Comitê de Ética em Pesquisa da Universidade Federal de São João del-Rei (UFSJ). Foi solicitado que os respondentes assinassem um Termo de Consentimento Livre e Informado, confirmando que estavam cientes dos objetivos do estudo e aceitavam participar. A participação de todos foi voluntária. 


\section{Resultados}

\section{Descrição das amostras}

As Tabelas 1 e 2 mostram os dados descritivos das amostras de pacientes e familiares. Os pacientes, em sua maioria, eram do sexo feminino (54\%), solteiros (43\%), com idade média de 39,87 anos (DP $\pm 11,03)$, sendo a mínima de 20 e máxima de 70 anos e possuíam o primeiro grau incompleto (64\%). Grande parte possuía renda própria (59\%), cuja principal origem era o auxílio-governo $(42,37 \%)$ e cujo valor era, na maioria dos casos (47\%), de um a dois salários mínimos. A maioria dos pacientes possuía diagnóstico de esquizofrenia, transtornos esquizotípicos e transtornos delirantes (54\%). A maioria não apresentava doença física (53\%) e não tinha apresentado nenhuma crise no último ano (58\%). A idade média de início do transtorno mental foi de 26,75 anos (DP $\pm 11,61$ ), a duração média da doença era de 13,08 anos (DP $\pm 10,00)$ e a duração média do tratamento era de 6,53 anos (DP $\pm 6,31)$. Exatamente a metade da amostra de pacientes nunca foi internada em hospitais psiquiátricos.

A idade média dos familiares era de 49,74 anos (DP $\pm 14,34$ ), sendo a mínima de 18 e a máxima de 78 anos. A maioria dos familiares era do sexo feminino (78\%), casado (51\%), com primeiro grau incompleto (63\%). A maioria possuía renda própria (61\%), de um a dois salários mínimos (78\%), originada principalmente de emprego ou trabalho regular (27\%) e de pensão (19\%). Com relação ao grau de parentesco, os pais/mães foram identificados como os principais cuidadores dos pacientes (39\%).

\section{Análises intragrupo da percepção de mudanças}

Na Tabela 3, são apresentadas as porcentagens de resposta de melhora, piora e ausência de mudanças, para cada item da escala EMP, bem como suas classificações pela análise da média de postos de Friedman, para cada grupo, separadamente. Os resultados mostram uma diferença significativa entre os escores de percepção de mudança entre os itens da escala, tanto no grupo de pacientes $\left(\chi^{2}=245,69 ; \mathrm{p}<0,0001\right)$, quanto no grupo dos familiares $\left(\chi^{2}=305,04 ; \mathrm{p}<0,0001\right)$.

As maiores porcentagens de melhora percebida por ambos os grupos e as maiores pontuações, na classificação de postos de Friedman, se concentraram principalmente nos itens: problemas pessoais, humor, sono e convivência com a família. Quanto à percepção de ausência de mudança, as porcentagens mais elevadas foram para os itens de convivência com os amigos, se- xualidade, capacidade de cumprir obrigações e atividades de lazer. No grupo de pacientes, o item apetite também obteve altos relatos de ausência de mudança.

Com relação à percepção de piora, as maiores porcentagens se concentraram nos itens sexualidade, energia e saúde física. O item sexualidade também obteve a menor pontuação na análise de postos de Friedman, indicando menor percepção de melhora pelos grupos.

\section{Análises intergrupos: comparação entre pacientes e familiares}

\section{- Comparação das classificações dos postos de Friedman}

Na última coluna da Tabela 3, encontra-se a ordem de classificação dos escores de percepção de mudanças dos pacientes e de seus familiares, pela análise das médias de postos de Friedman. Estes dados indicam em quais aspectos foram percebidos maiores graus de melhora, classificados em ordem. Pode-se observar que as médias de postos se distribuem de modo semelhante em ambos os grupos, sendo que as maiores e as menores médias de postos se concentraram nos mesmos itens da escala para os pacientes e para os seus familiares, indicando que eles se assemelham na ordem de suas percepções de mudança.

Foi feita uma análise correlacional de Spearman entre as médias de postos dos grupos de pacientes e de familiares. Os resultados mostram uma correlação positiva significativa $(\mathrm{p}<0,001)$ entre os escores dos dois grupos $(r=0,93)$. Quanto maiores eram os escores de melhora percebida, para cada item, por um grupo, maiores eram também estes valores para o outro grupo. Uma análise estatística adicional foi realizada com estes pares de escores, através do teste deWilcoxon, cujos resultados confirmaram que não há uma diferença estatisticamente significativa entre os valores das médias de postos dos dois grupos $(\mathrm{p}=0,84)$.

\section{- Comparação das medianas de percepção de mudança}

Os dados das medianas de percepção de mudança, de cada item da escala EMP, foram comparados, entre os pares de pacientes e familiares, através do teste de diferenças de Wilcoxon. Os resultados indicaram que não houve diferenças significativas $(p<0,05)$ entre as medianas dos pares de itens, o que significa que os pacientes e familiares perceberam, de forma semelhante, as mudanças ocorridas nos diversos aspectos da vida do paciente. Estes resultados das medianas 
Frequências, porcentagens, médias e desvios-padrão (DP) das características sócio-demográficas dos pacientes e familiares.

\begin{tabular}{|c|c|c|}
\hline Variáveis & $\begin{array}{c}\text { Pacientes } \\
\text { Frequência (\%) }\end{array}$ & $\begin{array}{c}\text { Familiares } \\
\text { Frequência (\%) }\end{array}$ \\
\hline \multicolumn{3}{|l|}{ Sexo } \\
\hline Feminino & $54(54,00)$ & $78(78,00)$ \\
\hline Masculino & $46(46,00)$ & $22(22,00)$ \\
\hline \multicolumn{3}{|l|}{ Estado civil } \\
\hline Solteiro & $43(43,00)$ & $14(14,00)$ \\
\hline Casado & $36(36,00)$ & $51(51,00)$ \\
\hline Separado ou divorciado & $11(11,00)$ & $7(7,00)$ \\
\hline Vivendo como casado & $3(3,00)$ & $7(7,00)$ \\
\hline Viúvo & $2(2,00)$ & $21(21,00)$ \\
\hline Não informaram & $5(5,00)$ & 0 \\
\hline \multicolumn{3}{|l|}{ Escolaridade } \\
\hline Analfabeto & $6(6,00)$ & $8(8,00)$ \\
\hline Ensino Fundamental incompleto & $64(64,00)$ & $63(63,00)$ \\
\hline Ensino Fundamental completo & $10(10,00)$ & $11(11,00)$ \\
\hline Ensino Médio incompleto & $4(4,00)$ & $4(4,00)$ \\
\hline Ensino Médio completo & $12(12,00)$ & $11(11,00)$ \\
\hline Ensino Superior incompleto & $2(2,00)$ & $2(2,00)$ \\
\hline Ensino Superior completo & $2(2,00)$ & 0 \\
\hline Mobral & 0 & $1(1,00)$ \\
\hline \multicolumn{3}{|l|}{ Renda própria } \\
\hline Sim & $59(59,00)$ & $61(61,00)$ \\
\hline Não & $41(41,00)$ & $39(39,00)$ \\
\hline \multicolumn{3}{|l|}{ Origem da renda } \\
\hline Emprego ou trabalho regular & $7(11,86)$ & $17(27,87)$ \\
\hline Bico ou atividade temporária & $3(5,08)$ & $2(3,28)$ \\
\hline Aposentadoria por tempo & $1(1,69)$ & $4(6,56)$ \\
\hline Aposentadoria por idade & $1(1,69)$ & $6(9,84)$ \\
\hline Aposentadoria por invalidez & $16(27,12)$ & $11(18,03)$ \\
\hline Pensão & $2(2,39)$ & $12(19,67)$ \\
\hline Pensão e aposentadoria & 0 & $6(9,84)$ \\
\hline Pensão e emprego & $1(1,69)$ & 0 \\
\hline Seguro & $1(1,69)$ & 0 \\
\hline Auxílio-doença & $25(42,37)$ & $3(4,92)$ \\
\hline Bolsas de Programas do Governo & $2(3,39)$ & 0 \\
\hline \multicolumn{3}{|l|}{ Valor da renda (salários mínimos) * } \\
\hline$<1$ & $8(13,56)$ & $5(8,20)$ \\
\hline $1-2$ & $47(79,66)$ & $49(80,31)$ \\
\hline $2,1-3$ & $3(5,08)$ & $5(8,20)$ \\
\hline$>4$ & $1(1,69)$ & $2(2,28)$ \\
\hline \multicolumn{3}{|l|}{ Parentesco com o paciente } \\
\hline Mãe ou Pai & & $39(39,00)$ \\
\hline Irmão(ã) & & $19(19,00)$ \\
\hline Cônjuge & & $22(22,00)$ \\
\hline Filho(a) & & $10(10,00)$ \\
\hline Outro & & $10(10,00)$ \\
\hline
\end{tabular}

* O valor do salário mínimo na época do estudo foi de $\mathrm{R} \$ 465,00$. 
Tabela 2

Frequências, porcentagens, médias e desvios-padrão (DP) das características do quadro clínico dos pacientes.

\begin{tabular}{|c|c|c|}
\hline Variáveis & Frequência (\%) & Média (DP) \\
\hline \multicolumn{3}{|l|}{ Categoria diagnóstica } \\
\hline Esquizofrenia, transtornos esquizotípicos e transtornos & $54(54,00)$ & \\
\hline \multicolumn{3}{|l|}{ delirantes transtornos do humor e afetivos } \\
\hline Transtornos neuróticos relacionados ao estresse & $34(34,00)$ & \\
\hline Somatoforme & $12(12,00)$ & \\
\hline \multicolumn{3}{|l|}{ Doenças físicas } \\
\hline Sim & $47(47,00)$ & \\
\hline Não & $53(53,00)$ & \\
\hline Idade de início da doença & & 26,75 anos $( \pm 11,61)$ \\
\hline Duração da doença & & 13,08 anos $( \pm 10,00)$ \\
\hline Duração do tratamento & & 6,53 anos $( \pm 6,31)$ \\
\hline \multicolumn{3}{|l|}{ Frequência de crises no último ano } \\
\hline Não teve & $58(58,00)$ & \\
\hline $1-5$ & $35(35,00)$ & \\
\hline $6-10$ & $2(2,00)$ & \\
\hline $15-20$ & $2(2,00)$ & \\
\hline 21 ou mais & $3(3,00)$ & \\
\hline \multicolumn{3}{|l|}{ Internações } \\
\hline Nunca & $50(50,00)$ & \\
\hline $1-5$ & $39(39,00)$ & \\
\hline $6-10$ & $6(6,00)$ & \\
\hline $11-15$ & $3(3,00)$ & \\
\hline Não souberam informar & $2(2,00)$ & \\
\hline Duração total das internações & & 1,62 meses $( \pm 1,50)$ \\
\hline Número de remédios & & $3,10( \pm 1,37)$ \\
\hline \multicolumn{3}{|l|}{ Tipo de medicação recebida } \\
\hline Apenas via oral & $76(76,00)$ & \\
\hline Apenas via intramuscular & $1(1,00)$ & \\
\hline Ambas & $23(23,00)$ & \\
\hline \multicolumn{3}{|l|}{ Frequência da medicação via oral } \\
\hline Diária & $98(98,99)$ & \\
\hline Semanal & $1(1,01)$ & \\
\hline \multicolumn{3}{|l|}{ Frequência da medicação intramuscular } \\
\hline Quinzenal & $6(25,00)$ & \\
\hline Mensal & $14(58,33)$ & \\
\hline Outra frequência & $4(16,67)$ & \\
\hline \multicolumn{3}{|l|}{ Adesão ao medicamento } \\
\hline Toma remédio sozinho & $59(59,00)$ & \\
\hline Aceita que lhe deem o remédio & $34(34,00)$ & \\
\hline Não aceita tomar a medicação & $4(4,00)$ & \\
\hline Às vezes aceita tomar & $3(3,00)$ & \\
\hline
\end{tabular}


Porcentagens de respostas dos pacientes e dos familiares e média de postos de Friedman dos dois grupos para cada item da Escala de Mudança Percebida (EMP).

\begin{tabular}{|c|c|c|c|c|c|c|c|c|}
\hline \multirow[t]{2}{*}{ Itens } & \multicolumn{2}{|c|}{ Pior do que antes (\%) } & \multicolumn{2}{|c|}{ Sem mudança (\%) } & \multicolumn{2}{|c|}{ Melhor do que antes (\%) } & \multicolumn{2}{|c|}{ Média de postos (\%) } \\
\hline & Pacientes & Familiares & Pacientes & Familiares & Pacientes & Familiares & Pacientes & Familiares \\
\hline 1. Problemas pessoais & - & - & 19,00 & 17,00 & 81,00 & 83,00 & 12,32 & 12,65 \\
\hline 2. Humor & 1,00 & 1,00 & 22,00 & 21,00 & 77,00 & 78,00 & 11,91 & 12,17 \\
\hline $\begin{array}{l}\text { 3. Estabilidade das } \\
\text { emoções }\end{array}$ & 9,00 & 7,00 & 38,00 & 41,00 & 53,00 & 52,00 & 9,54 & 9,78 \\
\hline 4. Confiança em si mesmo & 7,00 & 6,00 & 23,00 & 30,00 & 70,00 & 64,00 & 11,00 & 10,74 \\
\hline 5. Interesse pela vida & 7,00 & 7,00 & 28,00 & 28,00 & 65,00 & 65,00 & 10,63 & 10,78 \\
\hline $\begin{array}{l}\text { 6. Capacidade de suportar } \\
\text { situações difíceis }\end{array}$ & 8,00 & 7,00 & 29,00 & 34,00 & 63,00 & 59,00 & 10,32 & 10,26 \\
\hline 7. Apetite & 9,00 & 19,00 & 48,00 & 22,00 & 43,00 & 59,00 & 8,59 & 9,73 \\
\hline 8. Energia & 23,00 & 25,00 & 22,00 & 26,00 & 55,00 & 49,00 & 9,00 & 8,46 \\
\hline 9. Sono & 8,00 & 8,00 & 15,00 & 17,00 & 77,00 & 75,00 & 11,53 & 11,51 \\
\hline 10. Saúde física & 19,00 & 18,00 & 35,00 & 40,00 & 46,00 & 42,00 & 8,42 & 8,3 \\
\hline 11. Sexualidade & 26,00 & 26,00 & 48,00 & 58,00 & 26,00 & 16,00 & 6,45 & 5,53 \\
\hline $\begin{array}{l}\text { 12. Convivência com a } \\
\text { família }\end{array}$ & - & 3,00 & 35,00 & 25,00 & 65,00 & 72,00 & 10,92 & 11,55 \\
\hline $\begin{array}{l}\text { 13. Convivência com } \\
\text { amigos }\end{array}$ & 7,00 & 4,00 & 54,00 & 57,00 & 39,00 & 39,00 & 8,41 & 8,68 \\
\hline $\begin{array}{l}\text { 14. Convivência com as } \\
\text { outras pessoas }\end{array}$ & 4,00 & 5,00 & 43,00 & 48,00 & 53,00 & 47,00 & 9,74 & 9,35 \\
\hline 15. Interesse em se ocupar & 11,00 & 11,00 & 32,00 & 42,00 & 57,00 & 47,00 & 9,76 & 9,04 \\
\hline 16. Atividades de lazer & 8,00 & 10,00 & 46,00 & 45,00 & 46,00 & 45,00 & 8,92 & 9,04 \\
\hline 17. Tarefas de casa & 11,00 & 13,00 & 37,00 & 39,00 & 52,00 & 48,00 & 9,26 & 9,01 \\
\hline $\begin{array}{l}\text { 18. Capacidade de } \\
\text { cumprir obrigações }\end{array}$ & 2,00 & 4,00 & 43,00 & 45,00 & 55,00 & 51,00 & 10,04 & 9,81 \\
\hline 19. Item geral & 1,00 & - & 6,00 & 5,00 & 93,00 & 95,00 & 13,29 & 13,64 \\
\hline
\end{tabular}

confirmam, portanto, os dados descritos anteriormente, referentes à comparação das médias de postos.

\section{- Comparação dos escores brutos de percepção de mudança}

Calculou-se o ICC entre cada par de escores brutos de percepção de mudança dos dois grupos. Foram encontradas correlações positivas significativas $(\mathrm{p}<0,05)$ para 13 dentre os 19 itens da escala, indicando, mais uma vez, a concordância entre as percepções de mudança dos pacientes e dos seus familiares (Tabela 4). Os itens nos quais se observaram divergências entre as percepções dos pacientes e familiares se referem, em sua maioria, a aspectos subjetivos, tais como interesse pela vida e humor.

Um segundo tipo de análise foi realizado, calculando-se a diferença entre os escores brutos da percepção de mudança de pacientes e familiares, colocados lado a lado, para cada item da escala (Tabela 5). Esta análise permitiu calcular, para cada item, a porcentagem de pares que concordaram totalmente em suas respostas (diferença igual a zero; $\mathrm{P}=\mathrm{F}$, onde $\mathrm{P}$ : pacientes e $\mathrm{F}$ : familiares), a porcentagem de pares onde o paciente percebeu mais melhora do que o familiar $(\mathrm{P}>\mathrm{F})$ e a porcentagem de pares onde o inverso ocorreu $(\mathrm{F}>\mathrm{P})$.

Os resultados mostram maiores porcentagens de pares concordantes do que discordantes, concentrados na coluna $\mathrm{P}=\mathrm{F}$. Nota-se que de $50 \%$ a $90 \%$ dos pares concordam totalmente quanto ao grau de mudança percebida para os itens da escala. Os itens com maior acordo entre os pares de pacientes e familiares foram: percepção geral de mudança, sono, problemas pessoais, humor, convivência com a família e convivência com os amigos. Por outro lado, os itens com 


\section{Tabela 4}

Valores do coeficiente de correlação intraclasse (ICC) entre os escores brutos do grau de percepção de mudança dos pacientes e dos familiares e níveis de significância (p).

\begin{tabular}{lcc}
\hline Itens & ICC & Valor de p \\
\hline 1. Problemas pessoais & 0,12 & 0,12 \\
2. Humor & 0,12 & 0,11 \\
3. Estabilidade das emoções & 0,18 & 0,04 * \\
4. Confiança em si mesmo & 0,20 & 0,02 * \\
5. Interesse pela vida & 0,14 & 0,08 \\
6. Capacidade de suportar situações difíceis & 0,24 & 0,01 * \\
7. Apetite & 0,16 & 0,05 \\
8. Energia & 0,39 & 0,00 * \\
9. Sono & 0,42 & 0,00 * \\
10. Saúde física & 0,21 & 0,02 * \\
11. Sexualidade & 0,37 & 0,00 * \\
12. Convivência com a família & 0,29 & 0,00 * \\
13. Convivência com amigos & 0,42 & 0,00 * \\
14. Convivência com as outras pessoas & 0,17 & 0,05 \\
15. Interesse em se ocupar & 0,29 & 0,00 * \\
16. Atividades de lazer & 0,33 & 0,00 * \\
17. Tarefas de casa & 0,48 & 0,00 * \\
18. Capacidade de cumprir obrigações & 0,29 & 0,00 * \\
19. Item geral & 0,09 & 0,2 \\
\hline * 0 C & &
\end{tabular}

${ }^{*} p<0,05$.

Tabela 5

Porcentagem de pares em que as respostas dos pacientes foram iguais $(P=F)$, superiores $(P>F)$ ou inferiores $(P<F)$ às dos familiares, quanto ao grau de mudança percebida.

\begin{tabular}{|c|c|c|c|c|c|}
\hline Itens & $\begin{array}{c}-2 \\
P<F(\%)\end{array}$ & $\begin{array}{c}-1 \\
P<F(\%)\end{array}$ & $\begin{array}{c}0 \\
P=F(\%)\end{array}$ & $\begin{array}{c}1 \\
P>F(\%)\end{array}$ & $\begin{array}{c}2 \\
P>F(\%)\end{array}$ \\
\hline 1. Problemas pessoais & - & 14,00 & 74,00 & 12,00 & - \\
\hline 2. Humor & 1,00 & 15,00 & 68,00 & 16,00 & - \\
\hline 3. Estabilidade das emoções & 2,00 & 24,00 & 51,00 & 19,00 & 4,00 \\
\hline 4. Confiança em si mesmo & 3,00 & 14,00 & 62,00 & 17,00 & 4,00 \\
\hline 5. Interesse pela vida & 5,00 & 15,00 & 58,00 & 19,00 & 3,00 \\
\hline 6. Capacidade de suportar situações difíceis & 3,00 & 17,00 & 57,00 & 20,00 & 3,00 \\
\hline 7. Apetite & 6,00 & 22,00 & 50,00 & 16,00 & 6,00 \\
\hline 8. Energia & 6,00 & 13,00 & 55,00 & 19,00 & 7,00 \\
\hline 9. Sono & 2,00 & 10,00 & 77,00 & 6,00 & 5,00 \\
\hline 10. Saúde física & 5,00 & 20,00 & 50,00 & 17,00 & 8,00 \\
\hline 11. Sexualidade & 1,00 & 18,00 & 55,00 & 22,00 & 4,00 \\
\hline 12. Convivência com a família & - & 19,00 & 67,00 & 13,00 & 1,00 \\
\hline 13. Convivência com amigos & 1,00 & 18,00 & 64,00 & 17,00 & - \\
\hline 14. Convivência com as outras pessoas & 1,00 & 20,00 & 52,00 & 25,00 & 2,00 \\
\hline 15. Interesse em se ocupar & 3,00 & 14,00 & 58,00 & 20,00 & 5,00 \\
\hline 16. Atividades de lazer & 3,00 & 15,00 & 60,00 & 20,00 & 2,00 \\
\hline 17. Tarefas de casa & 2,00 & 14,00 & 62,00 & 20,00 & 2,00 \\
\hline 18. Capacidade de cumprir obrigações & - & 17,00 & 62,00 & 19,00 & 2,00 \\
\hline 19. Item geral & 1,00 & 5,00 & 90,00 & 4,00 & - \\
\hline
\end{tabular}


menor acordo entre os pares foram: apetite, saúde física, estabilidade das emoções e convivência com as outras pessoas.

Com relação aos dados de discordância (colunas 2, 3 e 5, 6), percebe-se que há uma maior concentração de pares discordantes com uma diferença de um ponto entre eles, do que de dois pontos. Ou seja, quando há discordância, ela ocorre mais frequentemente com uma diferença menor entre os escores, sendo muito poucos os casos de uma diferença de dois pontos. Dependendo do tipo de item, a direção desta discordância varia, ora sendo o familiar ora o paciente que apresentou uma melhor percepção de mudança. Os itens em que a percepção do familiar foi melhor do que a do paciente $(\mathrm{P}<\mathrm{F})$, na coluna $(-1)$, foram: estabilidade das emoções e apetite. Os itens em que a percepção do paciente foi melhor do que a do familiar $(\mathrm{P}>\mathrm{F})$, com um ponto positivo $(+1)$ foram: convivência com as outras pessoas e sexualidade.

Quanto às diferenças de dois pontos entre os grupos $(-2 \mathrm{e}+2)$, os itens onde houve maior concentração de desacordo, quando a percepção do familiar foi melhor do que a do paciente $(\mathrm{P}<$ F; -2), foram: apetite e energia. A percepção dos pacientes foi melhor do que a dos familiares $(\mathrm{P}>$ $\mathrm{F} ;+2)$ principalmente nos itens saúde física e energia.

Estes dados foram submetidos a uma análise estatística, visando verificar para quais itens as concordâncias encontradas entre pacientes e familiares eram significativas, através do CV disponível na função Crosstabs do SPSS. Os resultados deste teste indicaram que os dois grupos concordaram significativamente em suas respostas em 13 dos 19 itens da escala. Estes itens foram: confiança em si mesmo, apetite, energia, sono, saúde física, sexualidade, convivência com a família, convivência com amigos, convivência com as outras pessoas, interesse em se ocupar, atividades de lazer, tarefas de casa e capacidade de cumprir obrigações.

\section{Análise das respostas à questão} aberta da EMP

Os resultados da questão aberta da escala mostraram que a grande maioria dos pacientes (98\%) e dos familiares (96\%) respondeu que o tratamento ajudou os pacientes a se sentir melhor. Ao justificarem suas respostas, o aspecto mais relatado, por ambos os grupos, foi o fato do paciente ter ficado mais calmo/mais tranquilo, após ter iniciado o seu tratamento na instituição.

$\mathrm{O}$ segundo aspecto mais mencionado pelos pacientes foi que eles haviam melhorado devido à medicação. Outros aspectos mencionados, com frequência, foram a melhora percebida: no sono, na disposição física, nas alucinações/delírios e no fato de não ser mais errante. No grupo de familiares, os aspectos mais mencionados como tendo melhorado, em função do tratamento, foram as atividades desenvolvidas pelos pacientes, o seu sono, o fato de o paciente não ser mais errante e a sua disposição física. Em seguida, encontra-se a melhora atribuída à medicação, no apetite e na diminuição dos episódios de crise dos pacientes.

A análise das categorias de respostas mostrou uma grande similaridade entre pacientes e familiares. As mesmas categorias de análise emergiram dos relatos dos dois grupos, totalizando oito: (1) sintomas da doença e crises, (2) saúde física, (3) comportamentos problemáticos, (4) aspectos relacionados ao serviço e à medicação, (5) autonomia e/ou cuidados pessoais, (6) ocupação, (7) relacionamento social e (8) aspectos que não melhoraram.

\section{Discussão}

A ausência de estudos precedentes comparando a percepção de mudanças ocorridas na vida de pacientes psiquiátricos, sob o ponto de vista dos próprios pacientes e dos seus familiares cuidadores, limita o âmbito da discussão dos presentes resultados. Porém, torna-se uma oportunidade ímpar de inauguração deste campo de investigação a respeito das percepções dos usuários dos serviços de saúde mental no Brasil.

Os resultados mostraram que a maioria das mudanças percebidas pelos pacientes psiquiátricos foram semelhantes às dos seus familiares. Estes resultados positivos podem ser explicados pelo fato de o instrumento de medida enfocar principalmente fatos observáveis que ocorrem na vida do paciente. Os poucos itens que não apresentaram um grau de acordo significativo entre pacientes e familiares se referiam a aspectos menos acessíveis à observação por parte dos familiares. Um fator que pode ter contribuído para este resultado é o quadro clínico moderado dos pacientes da amostra, que pode ter favorecido uma maior aderência ao tratamento e, consequentemente, maior grau de melhora dos pacientes, o que contribuiria para a ocorrência de acordo entre pacientes e familiares quanto à percepção de melhora. Entretanto, é importante ressaltar que as percepções de piora e ausência de mudança para alguns itens da escala também apresentaram alto grau de acordo entre pacientes e familiares.

Estes resultados atestam a confiabilidade das respostas dos pacientes, ao fazer seu julgamento 
sobre os resultados do tratamento, confirmadas pelos seus familiares, em termos das mudanças percebidas em suas vidas. Convergem ainda com os dados obtidos por Awad \& Voruganti $25 \mathrm{e}$ por Hunter \& Cameron 26 , que também constataram a confiabilidade das respostas de pacientes psiquiátricos, em termos de sua estabilidade temporal.

Pacientes e familiares perceberam mais ocorrência de melhoras, em diversos aspectos da vida do paciente, em função do tratamento. Contudo, houve também relatos de percepção de piora e de ausência de mudança, o que indica que a escala EMP é uma medida sensível para avaliar os resultados do tratamento, uma vez que ela indica pontos positivos e negativos, contrariamente a outras medidas, tais como a de satisfação com o serviço, que tem sido considerada como pouco sensível 10.

Os resultados sobre a distribuição dos itens onde se concentraram as maiores porcentagens de melhora, piora e ausência de mudança em ambos os grupos, corroboram dados de outros estudos descritivos, realizados separadamente com uma amostra de pacientes 20 e com uma amostra de familiares 21 , utilizando a EMP. Nestes estudos, foi encontrada a mesma distribuição do grau de mudança percebida ao longo dos itens da escala.

Houve maior proporção de pacientes e familiares com percepção de melhora, no item referente aos problemas pessoais, associados aos sintomas da doença, nas entrevistas. Estes resultados poderiam se atribuídos aos efeitos do tratamento medicamentoso, tendo em vista que os pacientes entrevistados faziam parte de um ambulatório e recebiam, essencialmente, um tratamento medicamentoso. Um segundo aspecto percebido como melhora foi a qualidade do convívio familiar, que também pode ter sido resultante da ação dos psicofármacos, uma vez que estes contribuem para a diminuição dos comportamentos problemáticos e da sintomatologia dos pacientes. Segundo Barroso et al. 27, a sobrecarga dos familiares de pacientes psiquiátricos é mais elevada em relação à supervisão dos comportamentos problemáticos dos pacientes do que a outros aspectos do papel de cuidador, portanto, uma diminuição destes comportamentos poderia resultar em uma melhora no relacionamento entre o paciente e seus familiares.

Por outro lado, os itens que se referem à convivência com os amigos e outras pessoas apresentaram escores elevados de percepção de ausência de mudança em ambos os grupos, o que sugere a falta de relacionamentos dos pacientes fora do ambiente familiar. Alguns pacientes e familiares chegaram a se queixar, durante as entrevistas, de que os pacientes não tinham amigos, pois esses tinham se afastado após o início da doença.

As maiores concentrações de percepção de piora, em ambos os grupos, se localizaram nos itens sexualidade, saúde física e energia. Uma hipótese que poderia explicar estes resultados seria a presença de efeitos colaterais dos psicofármacos, que podem afetar estas áreas da vida dos pacientes. Estes dados parecem indicar que o tratamento medicamentoso tanto seria o responsável pelas pioras percebidas nestes aspectos, quanto pelas melhoras observadas na sintomatologia dos pacientes, tal como indicado acima. Resultados confirmando a percepção de piora e ausência de mudanças, com relação ao item sexualidade, foram obtidos por Perrault et al. 22 .

O grau de acordo obtido na presente pesquisa entre pacientes e familiares contrasta com resultados de outros estudos 12,13, que avaliaram a opinião destes dois grupos sobre outros aspectos dos serviços, como as prioridades dos resultados do tratamento e dos serviços prestados. Os autores relataram resultados majoritários de desacordo entre pacientes e familiares. Uma possível explicação é que quando o foco da avaliação é um conceito subjetivo referente ao futuro, como as avaliações de prioridades, as opiniões tendem a divergir. Ao contrário, ao avaliar experiências concretas vividas por estes avaliadores no presente, como a percepção de mudanças na vida dos pacientes, torna-se mais provável obter opiniões convergentes.

Os estudos que compararam pacientes e familiares com relação à satisfação com os serviços 14,15 chegaram a conclusões diferentes, dependendo do grau de desenvolvimento dos serviços ou do tipo de serviço avaliado (ambulatorial ou hospitalar). O estudo de Gigantesco et al. ${ }^{14}$, por exemplo, comparou a satisfação de pacientes hospitalizados e ambulatoriais com a dos seus familiares, concluindo que pacientes hospitalizados estavam mais satisfeitos do que os seus familiares com a participação no programa de tratamento. Comparando-se a satisfação dos pacientes ambulatoriais e seus familiares, foram encontrados altos níveis de satisfação em ambos os grupos e, portanto, alto grau de acordo entre eles, em concordância com dados de Ruggeri 10 . Uma das possíveis explicações é que o conceito de satisfação é muito amplo, englobando vários aspectos dos serviços, o que deixa margem a divergências, enquanto o conceito de mudanças percebidas é menos amplo, focado especificamente nos resultados do tratamento, em termos de melhora, piora e ausência de mudanças em aspectos pontuais da vida dos pacientes.

A análise da questão aberta da escala EMP confirmou a similaridade entre as respostas dos 
grupos de pacientes e familiares. Porém, foram observadas algumas particularidades em cada grupo, geralmente em relação a aspectos menos observáveis por um avaliador externo. Por exemplo, apenas no grupo de pacientes houve relatos explícitos de melhora na agressividade. Uma possível explicação é que os familiares talvez tenham incluído este aspecto, mas apenas implicitamente, quando relataram que os pacientes haviam ficado mais calmos, em função do tratamento. Em contrapartida, apenas os familiares mencionaram a melhora dos pacientes nos cuidados com a higiene, talvez porque dificuldades neste aspecto constituem um importante fator de sobrecarga sentida pelos familiares 27 . $\mathrm{O}$ fato de os pacientes não mencionarem este aspecto pode estar relacionado à sua sintomatologia, que envolve a deterioração da higiene e dos cuidados pessoais 28 . Os familiares também foram os únicos a relatar uma melhora na frequência das internações dos pacientes, talvez porque tenderam a falar de assuntos que extrapolavam as questões da entrevista, enquanto os pacientes tenderam mais a responder apenas o que lhes era perguntado.

Os familiares também relataram, com maior frequência, a melhora ocorrida no grau de ocupação dos pacientes. Talvez, esta melhora tenha contribuído para a diminuição do sentimento de sobrecarga dos familiares, o que fez com que eles ressaltassem esse aspecto com maior frequência. O estudo de Barroso et al. 27 constatou maior sobrecarga dos familiares com relação à frequência de tarefas de assistência cotidiana ao paciente, tais como auxiliá-lo na sua alimentação, transporte, compras e tarefas de casa.

Outra constatação foi a identificação de diferentes opiniões existentes, dentro de cada grupo, sobre um mesmo assunto. No grupo de familiares, o fato de o paciente ficar mais em casa foi visto por alguns como positivo, mas na percepção de outros familiares, o paciente sair mais de casa é que foi considerado favorável. Estas divergências podem se explicar, talvez, em função das particularidades existentes na relação familiar/ paciente, nas características da sintomatologia de cada paciente, na maior ou menor frequência de comportamentos problemáticos e na presença de outros fatores afetando a vida dos cuidadores, como outras pessoas necessitando de cuidados na família 27.

Este estudo possui limitações no que se refere à possibilidade de generalização dos seus resultados, tendo em vista que a amostra não foi selecionada aleatoriamente da população-alvo. Além disso, possíveis casos de abandono do serviço e de perdas de pacientes podem ter limitado a amostra aos casos com resultados mais positi- vos, o que talvez possa ter favorecido a ocorrência de acordos entre pacientes e familiares. Outra limitação do estudo é que apesar dos resultados apresentarem correlações estatisticamente significativas entre as percepções dos pacientes e familiares, os valores destas correlações foram baixos. Este fato pode diminuir o impacto dos resultados, mas ainda assim são resultados importantes, tendo em vista que, na literatura da área, tem sido observado frequentemente desacordos ou resultados não significativos, conforme citado acima, nos estudos que avaliaram satisfação ou prioridades.

Por se tratar de uma pesquisa de corte transversal, não foi possível avaliar a evolução da percepção de mudança ao longo do tempo, após o tratamento. Pesquisas futuras, do tipo longitudinal, poderiam acompanhar a evolução da percepção de mudança de pacientes e familiares, nas diferentes fases do tratamento, identificando a sequência das percepções de mudanças em diferentes aspectos da vida do paciente, em função do tratamento recebido. Somente um estudo prospectivo poderia fornecer informações mais fidedignas da percepção de mudanças de pacientes e familiares, nas diferentes fases do tratamento. Estudos futuros poderão igualmente avaliar a percepção de mudança, em função do tratamento em serviços que incluam intervenções psicossociais, além do tratamento medicamentoso. Aspectos da vida do paciente onde não foram observadas mudanças, neste estudo, poderiam apresentar resultados diferentes, em função das diferenças dos ingredientes ativos do tratamento.

\section{Conclusões}

Os resultados desta pesquisa apontam para a necessidade do desenvolvimento de intervenções focadas no controle dos sintomas físicos apresentados pelos pacientes psiquiátricos, pois a medicação utilizada provoca possíveis efeitos colaterais que afetam a sexualidade, a energia e a saúde física, dentre outros, tal como constatado pela percepção dos pacientes e dos seus familiares. Clínicos e profissionais de saúde mental devem estar mais atentos a estes efeitos, já que muitos deles se constituem como um dos principais fatores de abandono do tratamento e, consequentemente, de recaída 29 . Ao mesmo tempo, estratégias devem ser desenvolvidas para a adesão medicamentosa, importante para o controle da sintomatologia dos pacientes, bem como de um acompanhamento intensivo dos seus comportamentos problemáticos, principalmente nos momentos de crise 27. 
O presente estudo mostrou, ainda, a utilidade da avaliação das mudanças percebidas pelo paciente e pelo seu familiar, em identificar aspectos que não apresentaram melhora ou até mostraram uma piora e que, portanto, devem ser priorizados no tratamento e a necessidade de melhoria nas intervenções, em função destas necessidades, conforme aponta Mercier et al. 5 . Devido à alta frequência de relatos de ausência de mudança nos relacionamentos dos pacientes, fora do convívio familiar, conclui-se que há necessidade de desenvolvimento de intervenções voltadas para a socialização do paciente, proporcionando maior integração social, também para os pacientes de ambulatório, como no caso deste estudo.

A temática da percepção de mudanças pelos pacientes e pelos seus familiares se mostra, por-

\section{Resumo}

A necessidade de avaliação dos resultados do tratamento em serviços de saúde mental, na perspectiva dos usuários, tem sido destacada na literatura. O objetivo deste estudo foi comparar a avaliação dos pacientes e dos seus familiares, em termos das suas percepções de mudanças ocorridas na vida do paciente, em função do tratamento recebido. Para isso, a Escala de Mudança Percebida (EMP) foi aplicada em cem pacientes psiquiátricos e cem familiares, atendidos em serviços públicos de saúde mental. Os resultados mostraram uma alta porcentagem de acordo entre as percepções de mudança dos dois grupos, para a maioria dos itens da escala e para as respostas à questão aberta da escala. Estes resultados confirmaram dados de estudos precedentes que compararam a satisfação de pacientes e familiares em relação a outros aspectos dos serviços. A convergência entre as percepções de mudanças, encontrada no presente estudo, atesta a validade das respostas dos pacientes ao fazer um julgamento sobre os resultados do tratamento em serviços de saúde mental.

Saúde Mental; Serviços de Saúde; Resultado de Tratamento tanto, de grande importância para a avaliação dos efeitos do tratamento recebido em serviços de saúde mental, uma vez que integra diferentes percepções e expectativas na avaliação da eficácia do tratamento, conforme recomendações da literatura da área. A medida de percepção de mudança indica o alcance e os reais impactos do tratamento na vida do paciente, tal como destacado por Kazdin 30 e Mercier et al. 5, o que constitui um indicador de validade social das intervenções efetuadas e um indicador da validade clínica do tratamento. A realização periódica de estudos como este, em serviços de saúde mental, pode auxiliar na definição de intervenções mais focadas na problemática de cada paciente, incrementando a eficácia dos programas desenvolvidos 26,31 e a qualidade dos serviços de saúde mental 1 .

\section{Colaboradores}

C. S. Costa participou da elaboração do projeto, execução da coleta e análise de dados e redigiu o artigo. M. Bandeira e R. L. A. Cavalcanti colaboraram na elaboração do projeto de pesquisa e do delineamento do estudo, na análise e discussão dos resultados e na redação final do trabalho. J. D. Scalon contribuiu na análise de dados quantitativos e elaboração do artigo e das tabelas de resultados.

\section{Agradecimentos}

Aos pacientes e aos seus familiares, bem como aos profissionais dos serviços participantes que, gentilmente, colaboraram com a pesquisa. À Fundação de Amparo a Pesquisa de Minas Gerais (FAPEMIG) pelo financiamento. 


\section{Referências}

1. World Health Organization. The World Health Report 2001. Mental health: new understanding, new hope. Geneva: World Health Organization; 2001.

2. Donabedian A. Evaluating the quality of medical care. Milbank Mem Fund Q 1966; 44 Suppl: 166-203.

3. World Health Organization. Consumer's and caregiver's satisfaction with mental health services: a multisite study. Geneva: Division of Mental Health, World Health Organization; 1996.

4. Furtado JP, Campos RO. Participação, produção de conhecimento e pesquisa avaliativa: a inserção de diferentes atores em uma investigação em saúde mental. Cad Saúde Pública 2008; 24:2671-80.

5. Mercier L, Landry M, Corbiere M, Perreault M. Measuring client's perception as outcome measurement. In: Roberts AR, Yeager KR, editors. Evidence-based practice manual: research and outcome measures in health and human services. Oxford: Oxford University Press; 2004. p. 904-9.

6. Donabedian A. Quality assurance in heath care: consumer's role. Qual Health Care 1992; 1:247-51.

7. Fakhoury WKH, Kaiser W, Roder-Wanner UU, Priebe $S$. Subjective evaluation: is there more than one criterion? Schizophr Bull 2002; 28:319-27.

8. Center for Drug Evaluation and Research/Center for Biologics Evaluation and Research/Center for Devices and Radiological Health. Patient-reported outcome measures: use in medical product development to support labeling claims. Rockville: US Department of Health and Human Services, Food and Drug Administration; 2006.

9. McCabe R, Saidi M, Priebe S. Patient-reported outcomes in schizophrenia. Br J Psychiatry Suppl 2007; 50:s21-8.

10. Ruggeri M. Patients' and relatives' satisfaction with psychiatric services: the state of the art of its measurement. Soc Psychiatry Psychiatr Epidemiol 1994; 29:212-27.

11. Hansson L. Outcome assessment in psychiatric service evaluation. Soc Psychiatry Psychiatr Epidemiol $2001 ; 36: 244-8$.

12. Fischer EP, Shumway M, Owen RR. Priorities of consumers, providers, and family members in the treatment of schizophrenia. Psychiatr Serv 2002; 53:724-9.

13. Holley HL, Hodges P, Jeffers B. Moving psychiatric patients from hospital to community, views of patients, providers, and families. Psychiatr Serv 1998; 49:513-7.

14. Gigantesco A, Picardi A, Chiaia E, Balbib A, Morosini P. Patients' and relatives' satisfaction with psychiatric services in a large catchment area in Rome. Eur Psychiatry 2002; 17:139-47.

15. Vicente B, Vielma M, Jenner FA, Mezzina R, Lliapas I. Users' satisfaction with mental health services. Int J Soc Psychiatry 1993; 39:121-30.

16. Osinaga VLM. Estudo comparativo entre os conceitos de saúde e de doença mental e a assistência psiquiátrica, segundo portadores e familiares [Tese de Doutorado]. Ribeirão Preto: Universidade de São Paulo; 2004.
17. Bandeira M, Calzavara MGP, Costa CS, Cesari L. Avaliação de serviços de saúde mental: adaptação transcultural de uma medida da percepção dos usuários sobre os resultados do tratamento. J Bras Psiquiatr 2009; 58:107-14.

18. Bandeira M, Andrade MCR, Costa CS, Silva MA. Percepção dos pacientes sobre o tratamento em serviços de saúde mental: validação da Escala de Mudança Percebida. Psicol Reflex Crit; no prelo.

19. Cesari L, Bandeira M, Felício CB. Propriedades psicométricas da Escala de Mudança Percebida (EMP) - versão dos familiares. In: IV Congresso Brasileiro de Avaliação Psicológica e XIV Conferência Internacional de Avaliação Psicológica: Formas e Contextos. Campinas: Universidade São Francisco; 2009. p. 418.

20. Silva M, Bandeira M, Calzavara MGP. As mudanças percebidas pelo paciente em função do seu tratamento em serviços de saúde mental. In: VI Congresso de Produção Científica da UFSJ [CD-ROM]. São João del-Rei: Universidade Federal de São João del-Rei; 2007.

21. Mussi SV, Bandeira M. Fatores associados à percepção pelos familiares dos serviços de saúde mental. In: VIII Congresso de Produção Científica da UFSJ [CD-ROM]. São João del-Rei: Universidade Federal de São João del-Rei; 2009.

22. Perreault M, White ND, Fabres E, Landry M, Anestin AS, Rabouin D. Relationship between perceived improvement and treatment satisfaction among clients of a methadone maintenance program. Eval Prog Plann 2010; 33:410-7.

23. Bardin L. Análise de conteúdo. São Paulo: Edições $70 ; 1979$.

24. Caregnato RCA, Mutti R. Pesquisa qualitativa: análise de discurso versus análise de conteúdo. Texto \& Contexto Enferm 2006; 15:679-84.

25. Awad G, Voruganti LNP. Intervention research in psychosis: issues related to the assessment of quality of life. Schizophr Bull 2000; 26:557-64.

26. Cameron R, Hunter R. The Scottish Schizophrenia Outcomes Study: a new paradigm for utilising selfreport assessments from patients with schizophrenia. European Psychiatric Review 2008; 1:7-9.

27. Barroso SM, Bandeira M, Nascimento E. Sobrecarga de familiares de pacientes psiquiátricos atendidos na rede pública. Rev Psiquiatr Clín (São Paulo) 2007; 34:270-7.

28. American Psychiatric Association. DSM-IV: manual diagnóstico e estatístico de transtornos mentais. 4a Ed. Porto Alegre: Editora Artes Médicas; 1995.

29. Cordás TA, Laranjeiras M. Efeitos colaterais dos psicofármacos na esfera sexual. Rev Psiquiatr Clín (São Paulo) 2006; 33:168-73.

30. Kazdin AE. The meaning and measurement of clinical significance. J Consult Clin Psychol 1999; 67:332-9.

31. Contandriopoulos AP, Champagne F, Potvin L, Denis JO, Boyle P. Saber preparar uma pesquisa. São Paulo: Editora Hucitec/Rio de Janeiro: ABRASCO; 1994.

Recebido em 28/Mar/2010

Versão final reapresentada em 29/Out/2010

Aprovado em 28/Fev/2011 